\title{
Article
}

\section{Degenerate Derangement Polynomials and Numbers}

\author{
Minyoung Ma and Dongkyu Lim *(D) \\ Department of Mathematics Education, Andong National University, Andong 36729, Korea; mmy@anu.ac.kr \\ * Correspondence: dklim@anu.ac.kr
}

check for updates

Citation: Ma, M.; Lim, D. Degenerate Derangement Polynomials and

Numbers. Fractal Fract. 2021, 5, 59. https: / doi.org/10.3390/fractalfract 5030059

Academic Editors: Praveen Agarwal, Hari Mohan Srivastava, Taekyun Kim and Shaher Momani

Received: 10 May 2021

Accepted: 18 June 2021

Published: 22 June 2021

Publisher's Note: MDPI stays neutral with regard to jurisdictional claims in published maps and institutional affiliations.

Copyright: (c) 2021 by the authors. Licensee MDPI, Basel, Switzerland. This article is an open access article distributed under the terms and conditions of the Creative Commons Attribution (CC BY) license (https:/ / creativecommons.org/licenses/by/ $4.0 /)$.
Abstract: In this paper, we consider a new type of degenerate derangement polynomial and number, which shall be called the degenerate derangement polynomials and numbers of the second kind. These concepts are motivated by Kim et al.'s work on degenerate derangement polynomials and numbers. We investigate some properties of these new degenerate derangement polynomials and numbers and explore their connections with the degenerate gamma distributions for the case $\lambda \epsilon$ $(-1,0)$. In more detail, we derive their explicit expressions, recurrence relations, and some identities involving our degenerate derangement polynomials and numbers and other special polynomials and numbers, which include the fully degenerate Bell polynomials, the degenerate Fubini polynomials, and the degenerate Stirling numbers of the first and the second kinds. We also show that those polynomials and numbers are connected with the moments of some variants of the degenerate gamma distributions. Moreover, we compare the degenerate derangement polynomials and numbers of the second kind to those of Kim et al.

Keywords: degenerate derangement polynomials; degenerate derangement polynomials of the second kind; degenerate gamma distribution; degenerate Fubini polynomials; fully degenerate Bell polynomials; degenerate Stirling numbers

MSC: 11B83; 11B73; 60G50

\section{Introduction}

A derangement is a permutation with no fixed points. In other words, a derangement is a permutation of the elements of a set that leaves no elements in their original places. The number of derangements of a set of size $n$ is called the $n$-th derangement number and denoted by $d_{n}$. It was Pierre Rémonde de Motmort who initiated the study of counting derangements in 1708 (see [1]).

Carlitz was the first one who studied degenerate versions of some special polynomials and numbers, namely the degenerate Bernoulli polynomials and numbers and degenerate Euler polynomials and numbers. In recent years, the study of various degenerate versions of some special polynomials and numbers regained the interests of quite a few mathematicians and yielded many interesting arithmetical and combinatorial results. It is remarkable that the study of degenerate versions is not just limited to polynomials, but can be extended to transcendental functions such as gamma functions (see [2,3]).

As is well known, the generating function of the derangement numbers $d_{n}$ (see [1,4-8]) is given by

$$
\frac{1}{1-t} e^{-t}=\sum_{n=0}^{\infty} d_{n} \frac{t^{n}}{n !} .
$$

From (1), we note (see [6-9]) that

$$
d_{n}=n ! \sum_{i=0}^{n} \frac{(-1)^{i}}{i !}, \quad(n \geq 0) .
$$


The derangement polynomials $d_{n}(x)$ (see $\left.[7,8]\right)$ are defined by the generating function as

$$
\frac{1}{1-t} e^{(x-1) t}=\sum_{n=0}^{\infty} d_{n}(x) \frac{t^{n}}{n !}
$$

By (3), we get for $n \geq 0$,

$$
\begin{aligned}
d_{n}(x) & =\sum_{l=0}^{n}\left(\begin{array}{l}
n \\
l
\end{array}\right) d_{l} x^{n-l} \\
& =n ! \sum_{l=0}^{n} \frac{(x-1)^{l}}{l !}
\end{aligned}
$$

Clearly, we have $d_{n}(0)=d_{n}$.

For any nonzero real number $\lambda$, the degenerate exponential function $e_{\lambda}^{x}(t)($ see $[2,10-13])$ is defined as

$$
e_{\lambda}^{x}(t)=(1+\lambda t)^{\frac{x}{\lambda}}=\sum_{n=0}^{\infty} \frac{(x)_{n, \lambda}}{n !} t^{n}
$$

where $(x)_{0, \lambda}=1,(x)_{n, \lambda}=x(x-\lambda) \ldots(x-(n-1) \lambda),(n \geq 1)$. For brevity, we denote $e_{\lambda}^{1}(t)$ by $e_{\lambda}(t)$. From $(5)$, we can derive the inversion function of $e_{\lambda}(t)$ (see $\left.[6,14]\right)$ given by

$$
\log _{\lambda}(t)=\frac{1}{\lambda}\left(t^{\lambda}-1\right), \quad e_{\lambda}\left(\log _{\lambda}(t)\right)=t .
$$

In light of (3), Kim et al. [15] considered the degenerate derangement polynomials which are given by

$$
\frac{1}{1-t} e_{\lambda}^{x-1}(t)=\sum_{n=0}^{\infty} d_{n, \lambda}(x) \frac{t^{n}}{n !}
$$

When $x=0, d_{n, \lambda}=d_{n, \lambda}(0)$ are called the degenerate derangement numbers.

The degenerate Stirling numbers of the second kind (see [14]) given either by

$$
(x)_{n, \lambda}=\sum_{l=0}^{n} S_{2, \lambda}(n, l)(x)_{l}, \quad(n \geq 0),
$$

or by

$$
\frac{1}{m !}\left(e_{\lambda}(t)-1\right)^{m}=\sum_{n=m}^{\infty} S_{2, \lambda}(n, m) \frac{t^{n}}{n !}, \quad(m \geq 0),
$$

where $(x)_{0}=1,(x)_{n}=x(x-1) \ldots(x-n+1),(n \geq 1)$. either by

Recall that from $[3,14]$ that the degenerate Stirling numbers of the first kind are defined

$$
(x)_{n}=\sum_{l=0}^{n} S_{1, \lambda}(n, l)(x)_{l, \lambda}, \quad(n \geq 0),
$$

or by

$$
\frac{1}{m !}\left(\log _{\lambda}(1+t)\right)^{m}=\sum_{n=m}^{\infty} S_{1, \lambda}(n, m) \frac{t^{n}}{n !}, \quad(m \geq 0) .
$$

In this paper, we consider a new type of degenerate derangement polynomial and number, which shall be called the degenerate derangement polynomials and numbers of the second kind. These concepts are motivated by Kim et al.'s work on degenerate derangement polynomials and numbers in [15]. We investigate some properties of these new degenerate derangement polynomials and numbers and explore their connections with the degenerate gamma distributions for the case $\lambda \in(-1,0)$. In more detail, we derive their explicit expressions, recurrence relations, and some identities involving our degenerate derangement polynomials and numbers and other special polynomials and 
numbers, which include the fully degenerate Bell polynomials, the degenerate Fubini polynomials, and the degenerate Stirling numbers of the first and the second kinds. We also show that those polynomials and numbers are connected with the moments of some variants of the degenerate gamma distributions. Moreover, we compare the degenerate derangement polynomials and numbers of the second kind to those of Kim et al.

\section{Degenerate Derangement Polynomials of the Second Kind}

We try to degenerate the exponential function part of derangement polynomials in (3), slightly different way of (6). We consider the degenerate derangement polynomials of the second kind $d_{n, \lambda}^{*}(x)$, which are given by

$$
\frac{1}{1-t} e_{\lambda}((x-1) t)=\sum_{n=0}^{\infty} d_{n, \lambda}^{*}(x) \frac{t^{n}}{n !}
$$

When $x=0, d_{n, \lambda}^{*}=d_{n, \lambda}^{*}(0)$ are called the degenerate derangement numbers of the second kind.

From (5) and (7), we can express the $n$-th degenerate derangement polynomials of the second kind by the usual polynomials of degree $n$. This can be compared to the expression of the derangement polynomials $d_{n}(x)$ in (4).

$$
\begin{aligned}
\sum_{n=0}^{\infty} d_{n, \lambda}^{*}(x) \frac{t^{n}}{n !} & =\sum_{l=0}^{\infty} t^{l} \sum_{m=0}^{\infty}(x-1)^{m}(1)_{m, \lambda} \frac{t^{m}}{m !} \\
& =\sum_{n=0}^{\infty}\left(n ! \sum_{m=0}^{n} \frac{(x-1)^{m}(1)_{m, \lambda}}{m !}\right) \frac{t^{n}}{n !} .
\end{aligned}
$$

Comparing the coefficients on both sides of (8), we express the $n$-th degenerate derangement polynomials of the second kind, as the polynomials of degree $n$.

Proposition 1. For $n \geq 0$, we have

$$
d_{n, \lambda}^{*}(x)=n ! \sum_{l=0}^{n} \frac{(x-1)^{l}(1)_{l, \lambda}}{l !} .
$$

In particular, for $x=0$, we obtain

$$
d_{n, \lambda}^{*}=n ! \sum_{l=0}^{n} \frac{(-1)^{l}(1)_{l, \lambda}}{l !}=n ! \sum_{l=0}^{n} \frac{\langle-1\rangle_{l, \lambda}}{l !}
$$

where $\langle x\rangle_{0, \lambda}=1,\langle x\rangle_{n, \lambda}=n(x+\lambda) \ldots(x+(n-1) \lambda),(n \geq 1)$.

We can compare the expressions of the $n$-th degenerate derangement numbers of the second kind in Proposition 1 to the $n$-th derangement numbers in (2). Now, we observe that

$$
e_{\lambda}((x-1) t)=1+\sum_{n=1}^{\infty}\left(d_{n, \lambda}^{*}(x)-n d_{n-1, \lambda}^{*}(x)\right) \frac{t^{n}}{n !} .
$$

From (5) and (9), we have for $n \geq 1$ that

$$
(x-1)^{n}(1)_{n, \lambda}=d_{n, \lambda}^{*}(x)-n d_{n-1, \lambda}^{*}(x),
$$

and

$$
\langle-1\rangle_{n, \lambda}=d_{n, \lambda}^{*}-n d_{n-1, \lambda}^{*}
$$


In addition, by (7), we get

$$
d_{n, \lambda}^{*}(x)=\sum_{l=0}^{n}\left(\begin{array}{l}
n \\
l
\end{array}\right) d_{l, \lambda}^{*} x^{n-l}(1)_{n-l, \lambda,} \quad(n \geq 0) .
$$

Therefore, by (10) and (11), we obtain the identities, which can be compared to those in ([15], Theorem 2).

Theorem 1. The following identities hold true:

$$
\begin{array}{rr}
d_{n, \lambda}^{*}(x)=\sum_{l=0}^{n}\left(\begin{array}{l}
n \\
l
\end{array}\right) d_{l, \lambda}^{*} x^{n-l}(1)_{n-l, \lambda}, & (n \geq 0), \\
(x-1)^{n}(1)_{n, \lambda}=d_{n, \lambda}^{*}(x)-n d_{n-1, \lambda}^{*}(x), & (n \geq 1), \\
\langle-1\rangle_{n, \lambda}=d_{n, \lambda}^{*}-n d_{n-1, \lambda}^{*}, & (n \geq 1) .
\end{array}
$$

Replacing $t$ by $1-e_{\lambda}(t)$ in (7), we get

$$
\begin{aligned}
e_{\lambda}\left((x-1)\left(1-e_{\lambda}(t)\right)\right) & =e_{\lambda}(t) \sum_{l=0}^{\infty} d_{l, \lambda}^{*}(x) \frac{1}{l !}\left(1-e_{\lambda}(t)\right)^{l} \\
& =\sum_{m=0}^{\infty} \frac{(1)_{m, \lambda}}{m !} t^{m} \sum_{j=0}^{\infty} \sum_{l=0}^{j}(-1)^{l} d_{l, \lambda}^{*}(x) S_{2, \lambda}(j, l) \frac{t^{j}}{j !} \\
& =\sum_{n=0}^{\infty}\left(\sum_{j=0}^{n} \sum_{l=0}^{j}\left(\begin{array}{c}
n \\
j
\end{array}\right)(1)_{n-j, \lambda}(-1)^{l} d_{l, \lambda}^{*}(x) S_{2, \lambda}(j, l)\right) \frac{t^{n}}{n !} .
\end{aligned}
$$

Here $S_{2, \lambda}(n, l)$ fo $(n \geq l)$, are the degenerate Stirling numbers of the second kind. Alternatively, (12) is also given by

$$
\begin{aligned}
e_{\lambda}\left((x-1)\left(1-e_{\lambda}(t)\right)\right) & =\sum_{m=0}^{\infty}(x-1)^{m}(1)_{m, \lambda} \frac{1}{m !}\left(1-e_{\lambda}(t)\right)^{m} \\
& =\sum_{n=0}^{\infty}\left(\sum_{m=0}^{n}(x-1)^{m}\langle-1\rangle_{m, \lambda} S_{2, \lambda}(n, m)\right) \frac{t^{n}}{n !} .
\end{aligned}
$$

Therefore, by (12) and (13), we obtain the following theorem, which can be compared to ([15], Theorem 2).

Theorem 2. For $n \geq 0$, we have

$$
\sum_{j=0}^{n} \sum_{l=0}^{j}\left(\begin{array}{c}
n \\
j
\end{array}\right)(1)_{n-j, \lambda}(-1)^{l} d_{l, \lambda}^{*}(x) S_{2, \lambda}(j, l)=\sum_{j=0}^{n}(x-1)^{j}\langle-1\rangle_{j, \lambda} S_{2, \lambda}(n, j) .
$$

Recently, Kim et al. [12] introduced the degenerate Fubini polynomials $F_{n, \lambda}(y)$ as

$$
\frac{1}{1-y\left(e_{\lambda}(t)-1\right)}=\sum_{n=0}^{\infty} F_{n, \lambda}(y) \frac{t^{n}}{n !} .
$$


Note that $\lim _{\lambda \rightarrow 0} F_{n, \lambda}(y)=F_{n}(y)$ are the ordinary Fubini polynomials (see [16]). Replacing $t$ by $e_{\lambda}(t)-1$ in (7), we get

$$
\begin{aligned}
\frac{1}{2-e_{\lambda}(t)} e_{\lambda}\left((x-1)\left(e_{\lambda}(t)-1\right)\right) & =\sum_{l=0}^{\infty} d_{l, \lambda}^{*}(x) \frac{1}{l !}\left(e_{\lambda}(t)-1\right)^{l} \\
& =\sum_{n=0}^{\infty}\left(\sum_{l=0}^{n} S_{2, \lambda}(n, l) d_{l, \lambda}^{*}(x)\right) \frac{t^{n}}{n !} .
\end{aligned}
$$

In terms of (14), we note that (15) is also given by

$$
\begin{aligned}
& \frac{1}{2-e_{\lambda}(t)} e_{\lambda}\left((x-1)\left(e_{\lambda}(t)-1\right)\right) \\
& =\sum_{l=0}^{\infty} F_{l, \lambda}(1) \frac{t^{l}}{l !} \sum_{m=0}^{\infty}(x-1)^{m}(1)_{m, \lambda} \frac{1}{m !}\left(e_{\lambda}(t)-1\right)^{m} \\
& =\sum_{l=0}^{\infty} F_{l, \lambda}(1) \frac{t^{l}}{l !} \sum_{j=0}^{\infty} \sum_{m=0}^{j}(x-1)^{m}(1)_{m, \lambda} S_{2, \lambda}(j, m) \frac{t^{j}}{j !} \\
& =\sum_{n=0}^{\infty}\left(\sum_{l=0}^{n} \sum_{m=0}^{l}\left(\begin{array}{c}
n \\
l
\end{array}\right) F_{n-l, \lambda}(1)(x-1)^{m}(1)_{m, \lambda} S_{2, \lambda}(l, m)\right) \frac{t^{n}}{n !} .
\end{aligned}
$$

Therefore, by (15) and (16), we obtain the following theorem, which give the expression of the linear combination of the degenerate derangement polynomials of the second kind and the degenerate Stirling numbers of the second kind. This can be compared to those in ([15], Theorem 4).

Theorem 3. For $n \geq 0$, we have

$$
\sum_{l=0}^{n} S_{2, \lambda}(n, l) d_{l, \lambda}^{*}(x)=\sum_{l=0}^{n} \sum_{m=0}^{l}\left(\begin{array}{l}
n \\
l
\end{array}\right) F_{n-l, \lambda}(1)(x-1)^{m}(1)_{m, \lambda} S_{2, \lambda}(l, m) .
$$

Replacing $t$ by $\log _{\lambda}(1+t)$ in (14) with $y=1$, the following is known in ([15], (2.11))

$$
\begin{aligned}
\frac{1}{1-t} & =\sum_{l=0}^{\infty} F_{l, \lambda}(1) \frac{1}{l !}\left(\log _{\lambda}(1+t)\right)^{l} \\
& =\sum_{n=0}^{\infty}\left(\sum_{l=0}^{n} F_{l, \lambda}(1) S_{1, \lambda}(n, l)\right) \frac{t^{n}}{n !} .
\end{aligned}
$$

Writing the left-hand side of (17) differently, we have

$$
\begin{aligned}
\frac{1}{1-t} & =\left(\frac{1}{1-t} e_{\lambda}(-t)\right) e_{\lambda}^{-1}(-t) \\
& =\sum_{l=0}^{\infty} d_{l, \lambda}^{*} \frac{t^{l}}{l !} \sum_{m=0}^{\infty}\langle 1\rangle_{m, \lambda} \frac{t^{m}}{m !} \\
& =\sum_{n=0}^{\infty}\left(\sum_{l=0}^{n}\left(\begin{array}{c}
n \\
l
\end{array}\right) d_{l, \lambda}^{*}\langle 1\rangle_{n-l, \lambda}\right) \frac{t^{n}}{n !} .
\end{aligned}
$$

Therefore, by (7), (17) and (18), we obtain the following theorem.

Theorem 4. For $n \geq 0$, we have

$$
n !=\sum_{l=0}^{n} F_{l, \lambda}(1) S_{1, \lambda}(n, l)=\sum_{l=0}^{n}\left(\begin{array}{l}
n \\
l
\end{array}\right) d_{l, \lambda}^{*}\langle 1\rangle_{n-l, \lambda}=\sum_{l=0}^{n}\left(\begin{array}{l}
n \\
l
\end{array}\right) d_{l, \lambda}^{*}(x)(1-x)^{n-l}(1)_{n-l, \lambda} .
$$


By using Theorem 4 and ([15] Theorem 2.5), we can compare our degenerate derangement polynomials of the second kind to those of polynomials in [15].

Corollary 1. For $n \geq 0$, we have

$$
\begin{aligned}
\sum_{l=0}^{n}\left(\begin{array}{c}
n \\
l
\end{array}\right) d_{l, \lambda}^{*}\langle 1\rangle_{n-l, \lambda} & =\sum_{l=0}^{n}\left(\begin{array}{l}
n \\
l
\end{array}\right) d_{l, \lambda}^{*}(x)(1-x)^{n-l}(1)_{n-l, \lambda} \\
& =\sum_{l=0}^{n}\left(\begin{array}{l}
n \\
l
\end{array}\right) d_{l, \lambda}(1)_{n-l, \lambda}=\sum_{l=0}^{n}\left(\begin{array}{l}
n \\
l
\end{array}\right) d_{l, \lambda}(x)(1-x)_{n-l, \lambda} .
\end{aligned}
$$

Recently, Kim-Kim [10] considered the fully degenerate Bell polynomials given by

$$
e_{\lambda}\left(x\left(e_{\lambda}(t)-1\right)\right)=\sum_{n=0}^{\infty} \operatorname{Bel}_{n, \lambda}(x) \frac{t^{n}}{n !} .
$$

Replacing $t$ by $\log _{\lambda}(1+t)$ and $x$ by $x-1$ in (19), we get

$$
\begin{aligned}
e_{\lambda}((x-1) t) & =\sum_{m=0}^{\infty} \operatorname{Bel}_{m, \lambda}(x-1) \frac{1}{m !}\left(\log _{\lambda}(1+t)\right)^{m} \\
& =\sum_{m=0}^{\infty} \operatorname{Bel}_{m, \lambda}(x-1) \sum_{n=m}^{\infty} S_{1, \lambda}(n, m) \frac{t^{n}}{n !} \\
& =\sum_{n=0}^{\infty}\left(\sum_{m=0}^{n} \operatorname{Bel}_{m, \lambda}(x-1) S_{1, \lambda}(n, m)\right) \frac{t^{n}}{n !} .
\end{aligned}
$$

Obviously, (20) is also given by

$$
e_{\lambda}((x-1) t)=\sum_{n=0}^{\infty}(x-1)^{n}(1)_{n, \lambda} \frac{t^{n}}{n !} .
$$

Therefore, by (20) and (21), we obtain an identity

$$
(x-1)^{n}(1)_{n, \lambda}=\sum_{m=0}^{\infty} \operatorname{Bel}_{m, \lambda}(x-1) S_{1, \lambda}(n, m) .
$$

The following identity comes directly from (22).

$$
\operatorname{Bel}_{n, \lambda}(x-1)=\sum_{m=0}^{n}(1)_{m, \lambda}(x-1)^{m} S_{2, \lambda}(n, m) .
$$

We can see that (22) and (23) are mutual inversion formulae. We record these as theorem.

Theorem 5. For $n \geq 0$, we have

$$
(x-1)^{n}(1)_{n, \lambda}=\sum_{m=0}^{n} \operatorname{Bel}_{m, \lambda}(x-1) S_{1, \lambda}(n, m),
$$

and

$$
\operatorname{Bel}_{n, \lambda}(x-1)=\sum_{m=0}^{n}(1)_{m, \lambda}(x-1)^{m} S_{2, \lambda}(n, m) .
$$

When $x=2$ in Theorem 5, we obtain the known result in [15]. 
Corollary 2 ([15], Theorem 2.6). For $n \geq 0$, we have

$$
(1)_{n, \lambda}=\sum_{m=0}^{n} \operatorname{Bel}_{m, \lambda} S_{1, \lambda}(n, m),
$$

and

$$
\operatorname{Bel}_{n, \lambda}=\sum_{m=0}^{n}(1)_{m, \lambda} S_{2, \lambda}(n, m)
$$

Combining (7) and (20)

$$
\begin{aligned}
\sum_{n=0}^{\infty} d_{n, \lambda}^{*}(x) & =\frac{1}{1-t} e_{\lambda}((x-1) t) \\
& =\left(\sum_{j=0}^{\infty} j ! \frac{t^{j}}{j !}\right)\left(\sum_{l=0}^{\infty} \sum_{m=0}^{l} \operatorname{Bel}_{m, \lambda}(x-1) S_{1, \lambda}(l, m) \frac{t^{l}}{l !}\right) \\
& =\sum_{n=0}^{\infty}\left(\sum_{l=0}^{n} \sum_{m=0}^{l}\left(\begin{array}{l}
n \\
l
\end{array}\right)(n-l) ! \operatorname{Bel}_{m, \lambda}(x-1) S_{1, \lambda}(l, m)\right) \frac{t^{n}}{n !}
\end{aligned}
$$

Therefore, by (24) we obtain an expression for the $n$-th degenerate derangement polynomials of the second kind by the linear combination of the degenerate Bell polynomials and the degenerate Stirling numbers of the first kind.

Corollary 3. For $n \geq 0$, we have

$$
d_{n, \lambda}^{*}(x)=\sum_{l=0}^{n} \sum_{m=0}^{l}\left(\begin{array}{l}
n \\
l
\end{array}\right)(n-l) ! \operatorname{Bel}_{m, \lambda}(x-1) S_{1, \lambda}(l, m) .
$$

The following is observed in ([15], (2.16))

$$
\begin{aligned}
\frac{1}{1-t} & =e_{\lambda}^{-1}\left(\log _{\lambda}(1-t)\right)=\sum_{m=0}^{\infty}(-1)_{m, \lambda} \frac{1}{m !}\left(\log _{\lambda}(1-t)\right)^{m} \\
& =\sum_{m=0}^{\infty}(-1)_{m, \lambda} \sum_{n=m}^{\infty}(-1)^{n} S_{1, \lambda}(n, m) \frac{t^{n}}{n !}=\sum_{n=0}^{\infty}\left(\sum_{m=0}^{n}(-1)_{m, \lambda}(-1)^{n} S_{1, \lambda}(n, m)\right) \frac{t^{n}}{n !}
\end{aligned}
$$

From Theorem 4 and (25), we obtain

$$
n !=(-1)^{n} \sum_{m=0}^{n}(-1)_{m, \lambda} S_{1, \lambda}(n, m)=\sum_{m=0}^{n}\left(\begin{array}{c}
n \\
m
\end{array}\right) d_{m, \lambda}^{*}(x)(1-x)^{n-m}(1)_{n-m, \lambda} .
$$

We have the following identities, where the first identity comes from (25) and the second one from Theorem 5. Comparing Corollary 1 and (25), we obtain which can compare the degenerate derangement polynomials of the second kind to the degenerate derange polynomials in (6).

Corollary 4 . For $n \geq 0$, we have

$$
(-1)^{n} \sum_{m=0}^{n}(-1)_{m, \lambda} S_{1, \lambda}(n, m)=\sum_{m=0}^{n}\left(\begin{array}{c}
n \\
m
\end{array}\right) d_{m, \lambda}^{*}(x)(1-x)^{n-m}(1)_{n-m, \lambda}=\sum_{m=0}^{n}\left(\begin{array}{c}
n \\
m
\end{array}\right) d_{m, \lambda}(x)(1-x)_{n-m, \lambda} .
$$


Replacing $t$ by $\log _{\lambda}(1-t)$ and $x$ by $x-1$ in (19), we get

$$
\begin{aligned}
e_{\lambda}((x-1)(-t)) & =\sum_{k=0}^{\infty} \operatorname{Bel}_{k, \lambda}(x-1) \frac{1}{k !}\left(\log _{\lambda}(1-t)\right)^{k} \\
& =\sum_{k=0}^{\infty} \operatorname{Bel}_{k, \lambda}(x-1) \sum_{n=k}^{\infty} S_{1, \lambda}(n, k)(-1)^{n} \frac{t^{n}}{n !} \\
& =\sum_{n=0}^{\infty}\left(\sum_{k=0}^{n} \operatorname{Bel}_{k, \lambda}(x-1) S_{1, \lambda}(n, k)(-1)^{n}\right) \frac{t^{n}}{n !} .
\end{aligned}
$$

We remark that (26) is alternatively given by

$$
e_{\lambda}((x-1)(-t))=e_{-\lambda}^{-1}((x-1) t)=\sum_{n=0}^{\infty}(-1)_{n,-\lambda}(x-1)^{n} \frac{t^{n}}{n !} .
$$

Thus, from (26) and (27), we have for $n \geq 0$ the relation

$$
(-1)^{n} \sum_{k=0}^{n} \operatorname{Bel}_{k, \lambda}(x-1) S_{1, \lambda}(n, k)=(x-1)^{n}(-1)_{n,-\lambda} .
$$

In particular, $x=2$ in (28) we have ([15], Theorem 6).

Replacing $t$ by $1-e_{-\lambda}(t)$ and $x$ by $x-1$ in (7), we get

$$
\begin{aligned}
e_{-\lambda}^{-1}(t) e_{\lambda}\left((x-1)\left(1-e_{-\lambda}(t)\right)\right) & =\sum_{m=0}^{\infty} d_{m, \lambda}^{*}(x) \frac{(-1)^{m}}{m !}\left(e_{-\lambda}(t)-1\right)^{m} \\
& =\sum_{m=0}^{\infty} d_{m, \lambda}^{*}(x)(-1)^{m} \sum_{n=m}^{\infty} S_{2,-\lambda}(n, m) \frac{t^{n}}{n !} \\
& =\sum_{n=0}^{\infty}\left(\sum_{m=0}^{n} d_{m, \lambda}^{*}(x)(-1)^{m} S_{2,-\lambda}(n, m)\right) \frac{t^{n}}{n !} .
\end{aligned}
$$

An alternative expression of (29) is given by

$$
\begin{aligned}
e_{-\lambda}^{-1}(t) e_{\lambda} & \left((x-1)\left(1-e_{-\lambda}(t)\right)\right)=e_{-\lambda}^{-1}(t) e_{-\lambda}^{x-1}\left(e_{-\lambda}(t)-1\right) \\
& =\sum_{l=0}^{\infty}(-1)_{l,-\lambda} \frac{t^{l}}{l !} \sum_{m=0}^{\infty}(x-1)_{m,-\lambda} \frac{1}{m !}\left(e_{-\lambda}(t)-1\right)^{m} \frac{t^{m}}{m !} \\
& =\sum_{n=0}^{\infty}\left(\sum_{m=0}^{n}\left(\begin{array}{c}
n \\
m
\end{array}\right)(x-1)_{m,-\lambda} S_{2,-\lambda}(n, m)(-1)_{n-m,-\lambda}\right) \frac{t^{n}}{n !}
\end{aligned}
$$

From (29) and (30), we have for $n \geq 0$ that

$$
\sum_{m=0}^{n}(-1)^{m} d_{m, \lambda}^{*}(x) S_{2,-\lambda}(n, m)=\sum_{m=0}^{n}\left(\begin{array}{c}
n \\
m
\end{array}\right)(x-1)_{m,-\lambda} S_{2,-\lambda}(n, m)(-1)_{n-m,-\lambda} .
$$

Therefore, by (28) and (31), we obtain an expression of the linear combination of the degenerate derangement polynomials of the second kind which the coefficients of the degenerate Stirling numbers with $-\lambda$.

Theorem 6. For $n \geq 0$, we have

$$
\sum_{m=0}^{n}(-1)^{m} d_{m, \lambda}^{*}(x) S_{2,-\lambda}(n, m)=\sum_{m=0}^{n}\left(\begin{array}{l}
n \\
m
\end{array}\right)(x-1)_{m,-\lambda} S_{2,-\lambda}(n, m)(-1)_{n-m,-\lambda} .
$$


By (7), we get

$$
\frac{1}{1+t} e_{-\lambda}(t)=\sum_{m=0}^{\infty} d_{m,-\lambda}^{*}(-1)^{m} \frac{t^{m}}{m !}
$$

Replacing $t$ by $e_{-\lambda}(t)-1$ in (32), we get

$$
\begin{gathered}
e_{-\lambda}^{-1}(t)=e_{-\lambda}\left(e_{-\lambda}(t)-1\right)=\sum_{m=0}^{\infty} d_{m,-\lambda}^{*}(-1)^{m} \frac{1}{m !}\left(e_{-\lambda}(t)-1\right)^{m} \\
=\sum_{m=0}^{\infty} d_{m,-\lambda}^{*}(-1)^{m} \sum_{j=m}^{\infty} S_{2,-\lambda}(j, m) \frac{t^{j}}{j !} \\
=\sum_{j=0}^{\infty}\left(\sum_{m=0}^{j} d_{m,-\lambda}^{*}(-1)^{m} S_{2,-\lambda}(j, m)\right) \frac{t^{j}}{j !}
\end{gathered}
$$

Alternatively, (33) is also given by

$$
\begin{aligned}
e_{-\lambda}^{-1}(t) e_{-\lambda}\left(e_{-\lambda}(t)-1\right) & =\sum_{l=0}^{\infty}(-1)_{l,-\lambda} \frac{t^{l}}{l !} \sum_{m=0}^{\infty} \operatorname{Bel}_{m,-\lambda}(1) \frac{t^{m}}{m !} \\
& =\sum_{n=0}^{\infty}\left(\sum_{m=0}^{n}\left(\begin{array}{c}
n \\
m
\end{array}\right) \operatorname{Bel}_{m,-\lambda}(-1)_{n-m,-\lambda}\right) \frac{t^{n}}{n !} .
\end{aligned}
$$

Therefore, by (33) and (34), we obtain a relation including $d_{m,-\lambda}^{*}$ and $\operatorname{Bel}_{m,-\lambda}$.

Theorem 7. For $n \geq 0$, we have

$$
\sum_{m=0}^{n}(-1)^{m} d_{m,-\lambda}^{*} S_{2,-\lambda}(n, m)=\sum_{m=0}^{n}\left(\begin{array}{c}
n \\
m
\end{array}\right) \operatorname{Bel}_{m,-\lambda}(-1)_{n-m,-\lambda} .
$$

When we compare Theorem 7 and ([15], (2.33)) we have an identity, one is expressed by the degenerate derangement numbers of the second kind and the other is expressed by the degenerate derangement numbers.

Corollary 5. For $n \geq 0$, we have

$$
\sum_{m=0}^{n}(-1)^{m} d_{m,-\lambda}^{*} S_{2,-\lambda}(n, m)=\sum_{m=0}^{n}(-1)^{m} d_{m, \lambda} S_{2,-\lambda}(n, m) .
$$

From (33), we observe that

$$
\begin{aligned}
e_{-\lambda}\left(e_{-\lambda}(t)-1\right) & =e_{-\lambda}(t) \sum_{m=0}^{\infty} d_{m,-\lambda}^{*}(-1)^{m} \sum_{j=m}^{\infty} S_{2,-\lambda}(j, m) \frac{t^{j}}{j !} \\
& =\sum_{k=0}^{\infty}(1)_{k,-\lambda} \frac{t^{k}}{k !} \sum_{j=0}^{\infty}\left(\sum_{m=0}^{j} d_{m,-\lambda}^{*}(-1)^{m} S_{2,-\lambda}(j, m)\right) \frac{t^{j}}{j !} \\
& =\sum_{n=0}^{\infty}\left(\sum_{j=0}^{n} \sum_{m=0}^{n}\left(\begin{array}{c}
n \\
j
\end{array}\right)(1)_{n-j,-\lambda} d_{m,-\lambda}^{*}(-1)^{m} S_{2,-\lambda}(j, m)\right) \frac{t^{n}}{n !} .
\end{aligned}
$$

Accordingly, it follows that

$$
e_{\lambda}^{-1}\left(1-e_{-\lambda}(t)\right)=e_{-\lambda}\left(e_{-\lambda}(t)-1\right)=\sum_{n=0}^{\infty} \operatorname{Bel}_{n,-\lambda}(1) \frac{t^{n}}{n !} .
$$

Therefore, by (35) and (36), we obtain the following theorem. 
Theorem 8. For $n \geq 0$, we have

$$
\operatorname{Bel}_{n,-\lambda}=\sum_{j=0}^{n} \sum_{m=0}^{j}\left(\begin{array}{c}
n \\
j
\end{array}\right)(1)_{n-j,-\lambda}(-1)^{m} d_{m,-\lambda}^{*} S_{2,-\lambda}(j, m) .
$$

We have an identity, one is expressed by $d_{n, \lambda}^{*}$ and the other is $d_{n, \lambda}$. i.e., the degenerate derangement numbers of the second kind are by $-\lambda$ and the degenerate derangement numbers are by $\lambda$.

Corollary 6. For $n \geq 0$, we have

$$
\sum_{j=0}^{n} \sum_{m=0}^{j}\left(\begin{array}{c}
n \\
j
\end{array}\right)(1)_{n-j,-\lambda}(-1)^{m} d_{m, \lambda} S_{2,-\lambda}(j, m)=\sum_{j=0}^{n} \sum_{m=0}^{j}\left(\begin{array}{c}
n \\
j
\end{array}\right)(1)_{n-j,-\lambda}(-1)^{m} d_{m,-\lambda}^{*} S_{2,-\lambda}(j, m) .
$$

For $r \in \mathbb{N}$, we define the degenerate derangement polynomials of the second kind of order $r$ which are given by

$$
\frac{1}{(1-t)^{r}} e_{\lambda}((x-1) t)=\sum_{n=0}^{\infty} d_{n, \lambda}^{*(r)}(x) \frac{t^{n}}{n !} .
$$

When $x=0, d_{n, \lambda}^{*(r)}=d_{n, \lambda}^{*(r)}(0)$ are called the degenerate derangement numbers of the second kind of order $r$.

For the case $r=1, d_{n, \lambda}^{*}(x)=d_{n, \lambda}^{*(1)}(x)$ are the degenerate derangement polynomials of the second kind in (7). From (37), we note that

$$
\begin{aligned}
\sum_{n=0}^{\infty} d_{n, \lambda}^{*(r)}(x) \frac{t^{n}}{n !} & =\sum_{m=0}^{\infty}\left(\begin{array}{c}
r+m-1 \\
m
\end{array}\right) t^{m} \sum_{l=0}^{\infty}(x-1)^{l}(1)_{l, \lambda} \frac{t^{l}}{l !} \\
& =\sum_{n=0}^{\infty}\left(n ! \sum_{l=0}^{n} \frac{(x-1)^{l}(1)_{l, \lambda}}{l !}\left(\begin{array}{c}
r+n-l-1 \\
n-l
\end{array}\right)\right) \frac{t^{n}}{n !} .
\end{aligned}
$$

Comparing the coefficients on both sides of (38), we obtain the following theorem.

Theorem 9. For $n \geq 0$, we have

$$
d_{n, \lambda}^{*(r)}(x)=n ! \sum_{l=0}^{n} \frac{(x-1)^{l}(1)_{l, \lambda}}{l !}\left(\begin{array}{c}
r+n-l-1 \\
n-l
\end{array}\right) .
$$

In particular, for $x=0$, we have

$$
d_{n, \lambda}^{*(r)}=n ! \sum_{l=0}^{n} \frac{(-1)^{l}(1)_{l, \lambda}}{l !}\left(\begin{array}{c}
r+n-l-1 \\
n-l
\end{array}\right) .
$$

\section{Further Remarks}

Let $f(x)$ be the probability density function of the continuous random variable $X$, and let $g(x)$ be a real-valued function. Then the expectation of $g(X), E[g(X)]$ (see [17]), is defined by

$$
E[g(X)]=\int_{-\infty}^{\infty} g(x) f(x) d x
$$

A continuous random variable $X$, whose density function is given by

$$
f(x)=\left\{\begin{array}{cc}
\beta e^{-\beta x} \frac{(\beta x)^{\alpha-1}}{\Gamma(\alpha)}, & \text { if } x \geq 0 \\
0, & \text { if } x<0
\end{array}\right.
$$

for some $\beta>0$ and $\alpha>0$, is said to be the gamma random variable with parameters $\alpha, \beta$ and denoted by $X \sim \Gamma(\alpha, \beta)$. 
Let $X \sim \Gamma(1,1)$. Then, for all $t<1$, we have

$$
\begin{aligned}
E\left[e^{X t} \cdot e_{\lambda}(-t)\right] & =e_{\lambda}(-t) \int_{0}^{\infty} e^{x t} e^{-x} d x \\
& =\frac{1}{1-t} e_{\lambda}(-t)=\sum_{n=0}^{\infty} d_{n, \lambda}^{*} \frac{t^{n}}{n !} .
\end{aligned}
$$

Clearly, we also have

$$
E\left[e^{X t} e_{\lambda}(-t)\right]=\sum_{n=0}^{\infty}\left(\sum_{m=0}^{n}\left(\begin{array}{c}
n \\
m
\end{array}\right)\langle-1\rangle_{n-m, \lambda} E\left[X^{m}\right]\right) \frac{t^{n}}{n !} .
$$

Therefore, by (39) and (40), we obtain the following equations.

For $n \geq 0$, we have

$$
\sum_{m=0}^{n}\left(\begin{array}{c}
n \\
m
\end{array}\right)\langle-1\rangle_{n-m, \lambda} E\left[X^{m}\right]=d_{n, \lambda}^{*}
$$

and, more generally, we also have

$$
\sum_{m=0}^{n}\left(\begin{array}{c}
n \\
m
\end{array}\right)(x-1)^{n-m}(1)_{n-m, \lambda} E\left[X^{m}\right]=d_{n, \lambda}^{*}(x) .
$$

Unless otherwise stated, for the rest of this section, we assume that $\lambda \in(-1,0)$. We consider the degenerate gamma function $\Gamma_{\lambda}^{*}(x)$, which is initially defined for $0<\operatorname{Re}(s)<$ $-\frac{1}{\lambda}$ by the following integral

$$
\Gamma_{\lambda}^{*}(s)=\int_{0}^{\infty} e_{\lambda}(-t) t^{s-1} d t
$$

can be continued to a meromorphic function on $\mathbb{C}$, whose only singularities are simple poles at $s=0,-1,-2, \ldots,-\frac{1}{\lambda},-\frac{1}{\lambda}+1,-\frac{1}{\lambda}+2, \ldots$. We get this idea from $[2,3,15]$. Thus, by (41), we get

$$
\Gamma_{\lambda}^{*}(k)=\frac{\Gamma(k)}{(1)_{k+1,-\lambda}}, \quad\left(k \in \mathbb{N}, \lambda \in\left(-\frac{1}{k}, 0\right)\right),
$$

and, in particular, we have

$$
\Gamma_{\lambda}^{*}(1)=\frac{1}{1+\lambda} .
$$

A random variable $X=X_{\lambda}$ is said to have the degenerate gamma distribution with parameters $\alpha$ and $\beta,\left(-\frac{1}{\lambda}>\alpha>0, \beta>0\right)$, and denoted by $X \sim \Gamma_{\lambda}^{*}(\alpha, \beta)$, if its probability density function has the form

$$
f_{\lambda}(x)=\left\{\begin{array}{cc}
\frac{1}{\Gamma_{\lambda}^{*}(\alpha)} \beta(\beta x)^{\alpha-1} e_{\lambda}(-\beta x), & \text { if } x \geq 0, \\
0, & \text { otherwise. }
\end{array}\right.
$$

Note that $\frac{d}{d x} e_{-\lambda}^{c}(x)=c e_{-\lambda}^{c+\lambda}(x)$, for any constant $c$. Then, for $X \sim \Gamma_{\lambda}^{*}(1,1)$, we have

$$
\begin{aligned}
E\left[e_{\lambda}(-(t+\lambda) X)\right] & =(1+\lambda) \int_{0}^{\infty} e_{\lambda}(-(t+\lambda) x) e_{\lambda}(-x) d x \\
& =(1+\lambda) \int_{0}^{\infty} e_{-\lambda}^{t-1+\lambda}(x) d x=1+\lambda \frac{1}{1-t} e_{\lambda}(-t) e_{\lambda}^{-1}(-t) \\
& =(1+\lambda) \sum_{l=0}^{\infty} d_{l, \lambda}^{*} \frac{t^{l}}{l !} \sum_{m=0}^{\infty}(-1)^{m}(-1)_{m, \lambda} \frac{t^{m}}{m !} \\
& =\sum_{n=0}^{\infty}(1+\lambda) \sum_{l=0}^{n} d_{l, \lambda}^{*}(-1)^{m}(-1)_{m, \lambda}\left(\begin{array}{c}
n \\
l
\end{array}\right) \frac{t^{n}}{n !}
\end{aligned}
$$


Evidently, we also have

$$
\begin{aligned}
E\left[e_{-\lambda}^{t+\lambda}(X)\right]=E\left[e_{\lambda}(-(t+\lambda) X)\right] & =E\left[\frac{1}{1-\lambda X}(1-\lambda X)^{-\frac{t}{\lambda}}\right] \\
& =\sum_{n=0}^{\infty} E\left[\frac{1}{1-\lambda X}\left(\frac{-1}{\lambda} \log (1-\lambda X)\right)^{n}\right] \frac{t^{n}}{n !} .
\end{aligned}
$$

Therefore, from (42) and (43), we obtain the following theorem.

Theorem 10. For $X \sim \Gamma_{\lambda}^{*}(1,1)$, we have

$$
E\left[\frac{1}{1-\lambda X}\left(\frac{(-1)^{n}}{\lambda} \log (1-\lambda X)\right)^{n}\right]=(1+\lambda) \sum_{l=0}^{n} d_{l, \lambda}^{*}\langle 1\rangle_{n-l, \lambda}\left(\begin{array}{l}
n \\
l
\end{array}\right) .
$$

Now, we observe for $n \geq 0$ that

$$
(\log (1-\lambda X))^{n}=n ! \sum_{m=n}^{\infty} S_{1}(m, n) \frac{(-\lambda)^{m}}{m !} X^{m},
$$

where $S_{1}(n, m)$ are the Stirling numbers of the first kind, (see $\left.[18,19]\right)$. In turn, we have

$$
E\left[\frac{(-1)^{n}}{1-\lambda X}\left(\frac{1}{\lambda} \log (1-\lambda X)\right)^{n}\right]=(-1)^{n} \frac{n !}{\lambda^{n}} \sum_{m=n}^{\infty} S_{1}(m, n) \frac{(-\lambda)^{m}}{m !} E\left[\frac{X^{m}}{1-\lambda X}\right] .
$$

From Theorem 10 and (44), we have for $n \geq 0$ that

$$
\sum_{n=m}^{\infty} S_{1}(n, m) \frac{\lambda^{m}}{m !} E\left[\frac{X^{m}}{1-\lambda X}\right]=(1+\lambda) \frac{\lambda^{n}}{n !} \sum_{l=0}^{n} d_{l, \lambda}^{*}\langle 1\rangle_{n-l, \lambda}\left(\begin{array}{l}
n \\
l
\end{array}\right)
$$

where $X \sim \Gamma_{\lambda}^{*}(1,1)$. we have

For $X_{1}, X_{2}, \ldots, X_{r} \sim \Gamma(1,1)$, assume that $X_{1}, X_{2}, \ldots, X_{r}$ are independent. Then,

$$
\begin{aligned}
E\left[e^{\left(X_{1}+X_{2}+\ldots+X_{r}\right) t} e_{\lambda}((x-1) t)\right] & =E\left[e^{X_{1} t}\right] E\left[e^{X_{2} t}\right] \ldots E\left[e^{X_{r} t}\right] \cdot e_{\lambda}((x-1) t) \\
& =\underbrace{\left(\frac{1}{1-t}\right) \times\left(\frac{1}{1-t}\right) \times \ldots \times\left(\frac{1}{1-t}\right)}_{r-\text { times }} e_{\lambda}((x-1) t) \\
& =\sum_{n=0}^{\infty} d_{n, \lambda}^{*(r)}(x) \frac{t^{n}}{n !}
\end{aligned}
$$

Alternatively, (45) is given by

$$
\begin{aligned}
E & {\left[e^{\left(X_{1}+\ldots+X_{r}\right) t} e_{\lambda}((x-1) t)\right] } \\
& =\sum_{l=0}^{\infty} E\left[\left(X_{1}+\ldots+X_{r}\right)^{l}\right] \frac{t^{l}}{l !} \sum_{m=0}^{\infty}(x-1)^{m}(1)_{m, \lambda} \frac{t^{m}}{m !} \\
& =\sum_{n=0}^{\infty}\left(\sum_{l=0}^{n}\left(\begin{array}{l}
n \\
l
\end{array}\right) E\left[\left(X_{1}+\ldots+X_{r}\right)^{l}\right](x-1)^{n-l}(1)_{n-l, \lambda}\right) \frac{t^{n}}{n !} .
\end{aligned}
$$

By (45) and (46), we obtain for $n \geq 0$ the expression

$$
d_{n, \lambda}^{*(r)}(x)=\sum_{l=0}^{n}\left(\begin{array}{l}
n \\
l
\end{array}\right) E\left[\left(X_{1}+\ldots+X_{r}\right)^{l}\right](x-1)^{n-l}(1)_{n-l, \lambda} .
$$




\section{Conclusions}

Motivated from the work of Kim et al. in [15], in this paper, we have dealt with a new type of degenerate derangement polynomials, which are called the degenerate derangement polynomials of the second kind $d_{n, \lambda}^{*}(x)$. Our polynomials are a degenerate version of the derangement polynomials $d_{n}(x)$. We derived their explicit expressions, recurrence relations, and some identities involving those polynomials and numbers and other special polynomials and numbers such as the fully degenerate Bell polynomials, the degenerate Fubini polynomials and the degenerate Stirling numbers of both kinds. We also introduced the higher-order degenerate derangement polynomials of the second kind. We also made a connection between degenerate derangement polynomials and our polynomials by using degenerate Fubini numbers. Then, we explored the degenerate gamma distributions as a degenerate version of the gamma distributions for the case $\lambda \in(-1,0)$. We showed that the moments of distributions coming from some variants of degenerate gamma distributions are related to the degenerate derangement polynomials or the degenerate derangement numbers or the higher-order degenerate derangement polynomials. In the Corollaries 1,4 and 5, we compared our degenerate derangement polynomials of the second kind to those polynomials in [15].

Author Contributions: The authors contributed equally to this work. All authors have read and agreed to the published version of the manuscript.

Funding: The work of D.L. was partially supported by the National Research Foundation of Korea (NRF) grant funded by the Korean government (MSIT) NRF-2021R1C1C1010902.

Institutional Review Board Statement: Not applicable.

Informed Consent Statement: Not applicable.

Data Availability Statement: Not applicable.

Acknowledgments: The authors are thankful to the anonymous referees for their careful corrections to and valuable comments on the original version of this paper.

Conflicts of Interest: The authors declare no conflicts of interest.

\section{References}

1. Carlitz, L. The number of derangements of a sequence with given specification. Fibonacci. Quart. 1978, 16, 255-258.

2. Kim, T.; Kim, D.S. Note on the Degenerate Gamma Function. Russ. J. Math. Phys. 2020, 27, 352-358. [CrossRef]

3. Kim, T.; Kim, D.S.; Kwon, J.; Lee, H. A note on degenerate gamma random variables. Revista de Edu. 2020, 388, 39-44.

4. Jackson, D.M. Laguerre polynomials and derangements. Math. Proc. Camb. Philos. Soc. 1976, 80, 213-214. [CrossRef]

5. Joseph, A.W. A problem in derangements. J. Inst. Actuar. Stud. Soc. 1946, 6, 14-22. [CrossRef]

6. Kim, T.; Kim, D.S. Some identities on derangement and degenerate derangement polynomials. In Advances in Mathematical Inequalities and Applications; Trends Math; Birkhäuser/Springer: Singapore, 2018; pp. 265-277.

7. Kim, T.; Kim, D.S.; Jang, G.-W.; Kwon, J. A note on some identities of derangement polynomials. J. Inequal. Appl. 2018, 2018, 40. [CrossRef]

8. Kim, T.; Kim, D.S.; Kwon, H.-I.; Jang, L.-C. Fourier series of sums of products of $r$-derangement functions. J. Nonlinear Sci. Appl. 2018, 11, 575-590. [CrossRef]

9. Kim, T.; Kim, D.S.; Dolgy, D.V.; Kwon, J. Some identities of derangement numbers. Proc. Jangjeon Math. Soc. 2018, $21,125-141$.

10. Dolgy, D.V.; Kim, D.S.; Kim, T.; Kwon, J. On fully degenerate Bell numbers and polynomials. Filomat 2020, 34, 507-514. [CrossRef]

11. Khan, W.A. A new class of degenerate Frobenius-Euler-Hermite polynomials. Adv. Stud. Contemp. Math. (Kyungshang) 2018, 28, 567-576.

12. Kim, T.; Kim, D.S.; Jang, G.-W. A note on degenerate Fubini polynomials. Proc. Jangjeon Math. Soc. 2017, $20,52-531$.

13. Ma, Y.; Kim, T. A note on negative $\lambda$-binomial distribution. Adv. Differ. Equ. 2020, 2020, 569. [CrossRef]

14. Kim, D.S.; Kim, T. A Note on a New Type of Degenerate Bernoulli Numbers. Russ. J. Math. Phys. 2020, 27, 227-235. [CrossRef]

15. Kim, T.; Kim, D.S.; Lee, H.; Jang, L.-C. A note on degenerate derangement polynomials and numbers. Aims Math. 2021, 6, 6469-6481. [CrossRef]

16. Kilar, N.; Simsek, Y. A new family of Fubini type numbers and polynomials associated with Apostol-Bernoulli numbers and polynomials. J. Korean Math. Soc. 2017, 54, 1605-1621.

17. Ross, S.M. Introduction to Probability Models, 11st ed.; Elsevier/Academic Press: Amsterdam, The Netherlands, 2014. 
18. Sharma, S.K.; Khan, W.A.; Araci, S.; Ahmed, S.S. New construction of type 2 degenerate central Fubini polynomials with their certain properties. Adv. Differ. Equ. 2020, 2020, 587. [CrossRef]

19. Simsek, Y. Identities on the Changhee numbers and Apostol-type Daehee polynomials. Adv. Stud. Contemp. Math. (Kyungshang) 2017, 27, 199-212. 